Please quote as: Mrass, V. \& Peters, C. (2018): Digitale Wertschöpfung durch Crowd Services: Neue Formen des Kundensupports am Beispiel Mila und Swisscom. In: Robra-Bissantz, S. \& Lattemann, C. (Eds.), Digital Customer Experience - mit digitalen Diensten Kunden gewinnen und halten (Vol. Edition HMD). Wiesbaden, Germany:Springer. 


\title{
Digitale Wertschöpfung durch Crowd Services: Neue Formen des Kundensupports am Beispiel Mila und Swisscom ${ }^{1}$
}

\section{Volkmar Mrass, Christoph Peters}

\begin{abstract}
Zusammenfassung
Die digitale Transformation verändert gegenwärtig die Art und Weise, wie Arbeit erbracht und organisiert wird. Zwei Trends sind dabei besonders signifikant: Die „Plattform-Ökonomie“, mit CrowdworkingPlattformen als einer ,Spielart", und die „Sharing-Economy“. In diesem Beitrag zeigen wir am Beispiel der Crowdworking-Plattform Mila und ihres Kunden und 51-prozentigen Eigentümers Swisscom AG, wie Unternehmen die Crowd für Wertschöpfung einsetzen, ihren Kundensupport ausbauen und damit Vorteile für alle Beteiligten generieren können. Die Plattform Mila verbindet in einem neuartigen Ansatz das Paradigma der Crowd, insbesondere die Nutzung einer Vielzahl von Leistungserbringern, mit den Prinzipien der SharingEconomy, vor allem dem Einbezug der Kunden als Produzenten von Dienstleistungen. Basierend auf Experten-Tiefen-Interviews mit Verantwortlichen von Mila und Swisscom analysieren wir in diesem Beitrag dieses neuartige Arbeitssystem und leiten Handlungsempfehlungen für Unternehmen ab.
\end{abstract}

Crowdworking-Plattformen, Crowd Services, Kundensupport, Plattform-Ökonomie, Sharing Economy

\section{$1.1 \quad$ Einführung}

Die Digitalisierung verursacht gegenwärtig tiefgreifende Veränderungen auf allen Ebenen. Neben Auswirkungen auf Individuen und deren Freizeit verändert sie auch die Art und Weise, wie Arbeit erbracht wird. Organisationen wie Unternehmen passen gegenwärtig ihre Strategien an, um diesen Herausforderungen zu begegnen. Digitale Technologien sind dabei wichtige Treiber der Transformation der Arbeitswelt sowie von Produktivität und Wachstum [1]. Eine große Anzahl innovativer, digitaler neuer Geschäftsmodelle der letzten Jahre basiert dabei auf dem Plattformprinzip, häufig ist auch von „Plattformökonomie“ die Rede. Eine "Spielart" hiervon sind Crowdworking-Plattformen: Internetbasierte Plattformen, auf denen bezahlte Arbeiten mittels eines offenen Aufrufs an eine größere Anzahl an potenziellen Bearbeitern („Crowd“) ausgeschrieben werden; diese Plattformen übernehmen als Intermediäre dabei die Abwicklung, Koordination und Steuerung.

Gerade für Ökonomien, die historisch eine starke industrielle Basis haben - wie das bei Deutschland als größter Volkswirtschaft Europas und viertgrößter Volkswirtschaft der Welt der Fall ist - gilt es, sich rechtzeitig auf diese Entwicklung einzustellen, um nicht weiter Boden gegenüber im Bereich plattformbasierter Geschäftsmodelle führenden Nationen wie den USA zu verlieren. Die Wettbewerbsfähigkeit ganzer Länder hängt davon ab, wie erfolgreich sie dieser Entwicklung begegnen können [2]. Ausgehend von der Erkenntnis, dass auf technologischen Entwicklungen basierende neue Geschäftsmodelle langfristig nicht verhindert werden können „was digitalisiert werden kann, wird digitalisiert“ ([3], S. 70) - ist ein erster wichtiger Schritt hierzu eine intensive Untersuchung und ein tiefes Verständnis der Funktionsweise solcher Crowdworking-Plattformen. Die Anzahl der Unternehmen, die sich solcher CrowdworkingPlattformen zur Aufgabenerledigung bedienen, wächst, die Art der Arbeiten, die hierdurch erledigt werden können, ebenfalls [4]. Gut ein Jahrzehnt nach der Einführung und Prägung des Begriffes durch Jeff Howe im Jahr 2006 kann heute eine Vielzahl an Tätigkeiten entlang der

${ }^{1}$ Dieser Beitrag basiert auf einem Paper, das für den Track 8 der Multikonferenz Wirtschaftsinformatik (MKWI) 2018 angenommen wurde 
Wertschöpfungskette eines Unternehmens mit Hilfe der „Crowd“ abgewickelt werden [5].

Ein im deutschsprachigen Raum neuartiger Bereich in diesem Leistungsportfolio ist der der Crowd Services für den Kundensupport. Unternehmen bedienen sich dabei mittels CrowdworkingPlattformen als Intermediären einer Vielzahl an Bearbeitern, um Beratungs- und Vertriebsleistungen rund um ihre Produkte erbringen zu lassen. Dabei kommt es nicht zwingend zu einer „Kannibalisierung“ existierender Arbeit; es entstehen vielmehr neue Serviceangebote, die sich den Prinzipien der Sharing-Economy bedienen und für die betreffenden Unternehmen und ihre Kunden zusätzlichen Mehrwert schaffen. Ein interessantes Beispiel einer solchen Plattform ist Mila (www.mila.com) mit Sitz in Zürich sowie einem Deutschland-Standort in Berlin.

Das Unternehmen Mila bietet seinen Kunden - vornehmlich aus der Elektronik, Energieversorgungs- und Telekommunikationsbranche - Services mit Schwerpunkt auf technischer Unterstützung durch seine Crowd, insbesondere seiner sogenannten „Friends“. Bei diesen handelt es sich um technikaffine Individuen, die sich mit Produkten des jeweiligen Kundenunternehmens gut auskennen, dieses im Rahmen einer Prüfung durch Mila nachgewiesen haben und zumeist in ihrer Freizeit eine Art "technische Nachbarschaftshilfe" erbringen. Die Crowd der Mila Friends lässt sich nach der Zielgruppe des beauftragenden Unternehmens in weitere Sub-Communities einteilen. Anhand einer solchen, den Swisscom Friends der Crowdworking-Plattform Mila, gehen wir in diesem Beitrag auf eine neue Form des Kundensupports von Unternehmen wie diesem führenden Schweizer Telekommunikationsanbieter und die daraus ableitbaren Handlungsempfehlungen näher ein.

Unsere Einblicke und Erkenntnisse hierzu resultieren neben Gesprächen mit Experten aus Unternehmen (u.a. im Rahmen eines Workshops am 21. März 2017 in München) und Verbänden (u.a. zweier großer deutscher Gewerkschaften im Rahmen eines gemeinsamen Projektes), Beiträgen aus öffentlich zugänglichen Quellen (Presseberichten und -mitteilungen, u.a. von der Nachrichtenagentur Reuters), Brancheninformationen (u.a. von TechCrunch) und dem InternetAuftritt von Mila nicht zuletzt aus intensiven, ein- bis eineinhalbstündigen Tiefen-Interviews mit Verantwortlichen von Mila und Swisscom: So am 27. Januar 2017 mit Mila-CEO Christian Viatte (telefonisch) und am 14. Februar 2017 mit dem Swisscom-Head of Customer Field Service (und damit Chef von rund 1.450 Mitarbeiterinnen und Mitarbeitern) Francesco Castelletti (vor Ort bei Swisscom in Bern). Die Interviews wurden mittels eines semi-strukturierten Interview-Leitfadens geführt, der genügend Raum dafür ließ, bei Bedarf weitere Nachfragen zu stellen. Die Interviews wurden mit Einverständnis der Interviewten aufgezeichnet, anschließend transkribiert und ausgewertet. Da es sich hierbei um ein neuartiges Phänomen handelt, lag unser zentrales Erkenntnisinteresse darin, mittels der Case Study Methode [6] explorativ zu untersuchen, wie Mila als Crowdworking-Plattform das Angebot des Kundensupports von Swisscom erweitert, dieses Arbeitssystem [7] inklusive der Rollenverteilung zwischen Mila und Swisscom zu analysieren und basierend darauf erste Handlungsempfehlungen für Unternehmen abzuleiten.

Die zentrale Forschungsfrage lautet dementsprechend: „Wie erweitert die Crowd ServicePlattform Mila den Support für die Privatkunden von Swisscom und welche ersten Handlungsempfehlungen lassen sich daraus für Unternehmen ableiten?" Dieser Beitrag hat folgenden weiteren Verlauf: Nach dieser Einführung (Abschnitt 1) wird die Crowd ServicePlattform Mila inklusive deren Zusammenspiel mit Swisscom vorgestellt (Abschnitt 2). Es folgt eine Analyse dieses Arbeitssystems (Abschnitt 3) und die Darstellung der Vor- und Nachteile solcher Crowd Services (Abschnitt 4). Schließlich werden konkrete Handlungsempfehlungen für Unternehmen abgeleitet und Schlussfolgerungen für die Zukunft gezogen (Abschnitt 5).

\section{$1.2 \quad$ Neue Formen des Kundensupports}

Bei Mila handelt es sich um eine relativ, junge' Crowdworking-Plattform, die im Jahr 2013 als Spin-off des Softwareherstellers Coresystems in der Schweiz gegründet und im Jahr 2014 auch in Deutschland gestartet ist. Das Unternehmen verfügt über eine rund 4.000-köpfige Crowd (in der Mehrzahl in der Freizeit tätige Friends, in der Schweiz aber auch hauptberuflich tätige Profis), die etwa 4.000 Serviceaufträge pro Monat bearbeitet und in 150 Städten aktiv ist. Mila selbst hat 26 eigene Mitarbeiter, die sich auf die Standorte Zürich (Sitz) und Berlin verteilen. Ende 2015 
übernahm das Schweizer Telekommunikationsunternehmen Swisscom AG die Mehrheit an Mila. Mila vermittelt technik-affine Mitglieder der Crowd an Endkunden, die bei der Einrichtung ihrer Technik selbst nicht weiterkommen, ein technisches Problem haben oder schlichtweg nicht die notwendige Zeit aufbringen wollen, um sich damit zu beschäftigen. Der persönliche Kontakt zwischen diesen Friends von Mila und den Kunden der Plattform beziehungsweise der Unternehmen wird online vermittelt, dabei wird auf zeitliche Flexibilität hohen Wert gelegt. Die Reaktionszeit ist gering, gemäß Mila werden über 40 Prozent der Aufträge innerhalb einer Minute angenommen. Innerhalb von 10 Minuten sind es 70 Prozent, innerhalb einer Stunde über 90 Prozent und nach drei Stunden sind 98 Prozent der Aufträge verteilt. Eine Service-Anfrage eines Kunden wird an die geografisch relevante Crowd der Mila Friends in der Nähe (in der Regel in einem Umkreis von 30 Kilometern) ausgeschrieben, was eine zeitnahe Anreise und damit verbunden geringere Kosten ermöglicht. Derjenige Crowd Worker (Friend), der den Job zuerst annimmt, führt inn auch aus. Neben dem Zugang per PC gibt es seit Februar 2017 auch eine neue App für Smartphones mit integriertem Service-Bot und On-Demand-Funktion, die eine Buchung von Services zu einem vom Kunden bestimmten Wunschtermin inklusive der Möglichkeit, „sofort“ (i.d.R. 10 bis 60 Minuten) technische Unterstützung zu erhalten, beinhaltet.

Der Schwerpunkt der Services liegt im Bereich der Installation, der Problembehebung oder Produkterläuterung zu TV-Geräten, Computern und Notebooks, Internet/Netzwerken, mobilen Endgeräten, Audio/Hifi oder SmartHome-Anwendungen. Mila nennt für den jeweiligen Service Orientierungspreise, die Vereinbarung des endgültigen Preises erfolgt jedoch direkt zwischen dem Mila Friend und dem Kunden und kann davon abweichen. Weitere Leistungen sind in Abstimmung zwischen den beiden Vertragsparteien ebenfalls möglich. Die Buchung, Abwicklung und Steuerung der jeweiligen Services erfolgt über die Mila-Plattform als Intermediär und Vermittler zwischen der Crowd und den Kunden. Der geografische Schwerpunkt der erbrachten Services liegt gegenwärtig in der Schweiz und Deutschland.

Swisscom nutzt Mila als zusätzlichen Kanal zum eigenen Kundensupport sowie dem Point of Sale (POS) für die Erbringung von Services rund um die Produkte. Je nach Anlass und Ziel wird dabei zwischen den jeweiligen Vertriebskanälen der passende Service empfohlen: Erweist sich beispielsweise ein Problem vor Ort als zu komplex und nicht durch Mila Friends zu beheben, wird auf den regulären Swisscom Kundendienst verwiesen. Umgekehrt sind die Swisscom Friends in das Serviceangebot des Unternehmens als ein Zugangskanal integriert.

\subsection{Analyse des Arbeitssystems}

Das Zusammenspiel der Beteiligten auf Seiten der Crowdworking-Plattform Mila, deren Crowd, Swisscom sowie deren Endkunden lässt sich gut als Arbeitssystem anhand Alter's Work System Frameworks [7] analysieren. Zudem lässt sich am Beispiel Mila und ihres Kunden (und zugleich 51-prozentigen Eigentümers) Swisscom gut aufzeigen, wie Unternehmen heute das Prinzip der "Wisdom of Crowds" [8] nutzen können, um ihren Kundensupport auszubauen. Und dabei zugleich dem Trend der On-Demand-Economy, die je nach Bedarf kurzfristig zu erbringende Services erfordert, sowie der Sharing-Economy, die Kunden zugleich zu Konsumenten und Produzenten („Prosumenten“) macht und ungenutzte Ressourcen teilt, zu begegnen. Für die Kunden von Swisscom erfolgt durch die Bereitstellung der in Abschnitt 2 vorgestellten

Beauftragungsmöglichkeiten über die Internetauftritte von Mila und Swisscom sowie über die Mobile App eine Erweiterung ihres Supports. Sie erhalten damit weitere Kommunikationskanäle und digitale Zugangsmöglichkeiten zum Unternehmen. Das Unternehmen Swisscom gewinnt auf Grund dieses Angebotes auch insbesondere junge, technikaffine Kundengruppen hinzu, die die Abwicklung von Services über digitale Kanäle auch bereits aus anderen Lebensbereichen kennen. Zugleich bedeutet dieses erweiterte digitale Angebot auch für die Teil-Kundengruppe der Swisscom Friends eine Erweiterung ihrer Rolle, da diese nun neben ihrer Funktion als Konsumenten der Produkte und Services von Swisscom gleichzeitig auch zu Mitwirkenden am Leistungserstellungsprozess werden. Letzterer wird für die Swisscom Friends ebenfalls digital über die Crowdworking-Plattform Mila koordiniert.

Auf Basis einer Analyse des Arbeitssystems („Work System“) der Crowdworking-Plattform Mila 
mittels des Work System Frameworks von ([7], S. 78) ermitteln wir im Folgenden nun Vor- und Nachteile der Nutzung solcher Crowd Services. Wir wählen dieses Framework und die dahinterliegende Work System Theorie von Alter (2013) als Instrumente, da beide eine sehr gute Basis für die Analyse des Zusammenspiels zwischen Teilnehmenden (vor allem Mila Friends, teilweise aber auch die Kunden), Information (beispielsweise das Wissen über SwisscomProdukte) und Technologien (beispielsweise die Mila Mobile App) zur Durchführung der Prozesse und Aktivitäten (beispielsweise Installation, Problembehebung und Produktberatung) bieten. Das Work System Framework ermöglicht insbesondere auch die Analyse von soziotechnischen Systemen wie demjenigen, in das Mila und Swisscom eingebunden sind.

Um die Analyse zu strukturieren, führen wir diese und die Ermittlung der Vor- und Nachteile entlang der internen Kernelemente des Work System Frameworks Prozesse und Aktivitäten, Teilnehmende, Information und Technologien durch (Infrastruktur, Strategien und Umwelt bilden die für jedes Unternehmen bestehenden Rahmenbedingungen und sich nicht Teil des Work Systems). Sowie zusätzlich auch entlang der in diesem Fall ebenfalls innerhalb des Work Systems befindlichen Kunden (da Kunden hier gleichzeitig auch Teilnehmende sind, die bei der ServiceErbringung mitwirken und die Services erst im Zusammenspiel zwischen Crowd und Kunden Gestalt annehmen) und Produkte \& Services (siehe Abbildung 1). Basierend auf den ermittelten Vor- und Nachteilen und zusätzlich unseren in Gesprächen mit Experten gewonnenen Erkenntnissen leiten wir im Anschluss daran konkrete Handlungsempfehlungen für Unternehmen ab.

\section{Platzhalter Abbildung Start}

Abb. 1 Darstellung des Arbeitssystems/Work Systems (fett markierter Bereich) von Mila und dem Kunden Swisscom in Anlehnung an Alter's Work System Frameworks (Quelle: eigene Darstellung in Anlehnung an [7], S. 78) ${ }^{2}$

Datei: Abbildung 1_Autorenbeitrag Mrass_Peters.tif

Abb.-Typ: Halbton-Abb.

Farbigkeit (IST): 1c

Farbigkeit (SOLL): 1c

Bildrechte: [Urheberrecht beim Autor]

Abdruckrechte: Nicht notwendig

Hinweise Verlag/Setzerei:

Platzhalter Abbildung Stop

\subsection{Crowd Kundensupport: Vor- und Nachteile der Nutzung}

Der Ausbau des Kundensupports mittels Crowd Services und Intermediären wie der Crowdworking-Plattform Mila birgt für Unternehmen Vor- und Nachteile, die hier nun stichpunktartig dargestellt und entlang der Elemente des Arbeitssystems/Work System Frameworks gegliedert und erläutert werden.

Kunden. An der Spitze des Work System Frameworks und im Mittelpunkt der Anstrengungen von Unternehmen stehen die Kunden. Die Kunden von Swisscom erhalten auf Grund der großen Anzahl an möglichen Bearbeitern aus der Mila-Crowd und der Tatsache, dass diese auch abends und an Wochenenden zur Verfügung stehen, kurzfristig Unterstützung. In der Regel wird ein Auftrag - siehe Abschnitt 2 - spätestens innerhalb von 3 Stunden angenommen. Mittels der Smartphone-App besteht zudem die Möglichkeit der Buchung von kurzfristigen Services, bei denen eine technische Unterstützung innerhalb von 10 bis 60 Minuten erfolgt. Ein wesentlicher Vorteil für die Kunden im Vergleich zu von internen Mitarbeitern erbrachten Services ist also die Geschwindigkeit der Service-Erbringung. Swisscom kann seinen Kunden mittels der Crowd 
Services auch zu Zeiten anbieten, die außerhalb der regulären Kundendienstzeiten liegen. Aus der Tatsache, dass die Mila Friends in der Regel aus kurzer Entfernung anreisen und diese Services meist nebenher in ihrer Freizeit erbringen, resultiert ein weiterer Vorteil für die Kunden von Swisscom: Im Vergleich zu den durch interne Mitarbeiter von Swisscom erbrachten Services sind die Kosten in diesem Fall für sie deutlich geringer.

Neben diesen Vorteilen, die Unternehmen ihren Kunden bieten können, beinhaltet der Einsatz der Crowd auch Nachteile: Die Einheitlichkeit des Auftritts beim Kunden - Stichwort „Corporate Identity“ - lässt sich für Unternehmen mit eigenen Beschäftigten in der Regel besser gewährleisten als mit einer relativ heterogeneren externen Crowd. Interne Mitarbeiter sind zudem mit der Kultur und Vorgehensweise eines Unternehmens besser vertraut als externe Crowd Worker und können damit vor Ort beim Kunden stärker im Sinne des Unternehmens handeln.

Produkte \& Services. Zentrales Ergebnis der Anstrengungen und Tätigkeiten von Unternehmen sind Produkte und Services. Swisscom augmentiert mittels der Mila Crowd, die über ein hohes technisches Wissen verfügt, die bisherigen Services rund um Produkte wie Internet, Smartphones, Tablets, TV oder Wearables. Das Unternehmen verfügt mittels Mila Friends über einen weiteren digitalen Zugangskanal, über den Services rund um diese Produkte beauftragt werden können. Wie oben bereits geschildert, sind die Kosten für diese Services geringer als bei der Leistung durch interne Mitarbeiter. Damit ist dieses Angebot nach Angabe der Verantwortlichen von Mila und Swisscom auch für Kunden attraktiv, die nicht bereit sind, für diese Art von Services die regulär anfallenden Preise zu bezahlen. Gerade für Tätigkeiten, die - durch die hauptamtlichen Beschäftigten eines Unternehmens erbracht - relativ aufwändig und teuer wären und daher von Kunden eher gemieden werden, wird eine bestehende Lücke im Service-Angebot geschlossen. Swisscom als Unternehmen mit Wurzeln in der Schweiz kann zudem durch Einbindung der Crowd auch Services für seine Produkte in Ländern anbieten, in denen es nicht oder nur in sehr geringem Umfang vertreten ist und damit die Präsenz seiner Marke erhöhen. Das Angebot einer neuen Kundenschnittstelle, über die Serviceaufträge rund um die Produkte getätigt werden können, und die geografische Ausweitung des Vertriebsgebietes sind also weitere Vorteile für Unternehmen bei der Nutzung dieses Modells.

Auf der anderen Seite werden Crowd Worker/Friends, obwohl keine Angestellten des Unternehmens, sondern selbständig tätig, bei der Erbringung der Services dennoch oft als Unternehmensrepräsentanten wahrgenommen. Das kann sich insbesondere bei Fehlern bei der Erbringung der Services, mangelnder Kompetenz oder Unfreundlichkeit der Crowd Worker negativ auf die Kundenzufriedenheit und damit die Marke und das Unternehmen auswirken. Das Unternehmen hat hierauf jedoch gleichzeitig nur begrenzte Einfluss- und Steuerungsmöglichkeiten. Die Erbringung der Services durch externe Crowds kann außerdem zu einem Verlust an potenziellem Umsatz für das jeweilige Unternehmen führen. Das gilt insbesondere, wenn es sich nicht um Services handelt, die weitgehend komplementär sind, weil es zu den regulären Preisen und Konditionen des Kundendienstes des jeweiligen Unternehmens keinen Bedarf/Markt gäbe. Wenn es sich also um Services handelt, die der Kunde auch von internen Mitarbeitern des Unternehmens beziehen könnte, entgeht dem Unternehmen ein Teil des potenziellen Umsatzes.

Prozesse \& Aktivitäten. Die Erbringung der Leistungen eines Unternehmens ist mit Prozessen und Aktivitäten verbunden, für deren zustande kommen wiederum Teilnehmende, Informationen und Technologien notwendig sind. Im Kontext dieser Prozesse und Aktivitäten ist bezogen auf Mila festzustellen, dass ein vermittelter „Friend“ auf Grund der durch die Plattform gesteuerten Auswahl nach räumlicher Nähe zum Kunden lange Anfahrtswege vermeidet. Dies erhöht die Effizienz und Schnelligkeit der Leistungserbringung. Gleichzeitig erfolgt eine passgenauere Konzentration auf die jeweiligen lokalen Gegebenheiten. Daraus lässt sich der Vorteil ableiten, dass Unternehmen ihre Prozesse und Aktivitäten zur Erbringung von Services durch Einbindung der Crowd beschleunigen, effizienter gestalten und zielgerichteter auf die Erfordernisse des Marktes und lokale Erfordernisse ausrichten können.

Andererseits sind die Kontinuität und Verfügbarkeit einer gut qualifizierten Crowd für die Abwicklung der Prozesse und Aktivitäten essenziell. Crowdworking-Plattformen als Intermediäre 
können diese aber nicht garantieren. In Zeiten, in denen die Crowd beispielsweise durch den Einsatz für andere Unternehmen stark gefordert ist, mag die Arbeit für das eigene Unternehmen leiden. Auch der umgekehrte Fall, dass sich Mitglieder der Crowd abwenden, da nicht genügend Aufträge eingehen, und ihre Arbeitskraft lieber anderen Crowdworking-Plattformen und den sie beauftragenden Unternehmen zur Verfügung stellen, ist möglich.

Teilnehmende. Bei Teilnehmenden handelt es sich um Personen (oder auch Maschinen), welche die Arbeit innerhalb eines Arbeitssystems erbringen. Neben den Kunden von Swisscom, die an der Erbringung der Services mitwirken und daher zu den Teilnehmenden gehören, sind das hier insbesondere die Mila Friends. Diese sind selbständig tätig und nicht bei Mila oder Swisscom angestellt. Für die Mila Friends fallen also auf Seiten von Mila und Swisscom keine Löhne oder Sozialabgaben an, da deren Bezahlung (und damit indirekt auch die darin enthaltene Provision für die Vermittlungsplattform) von den Kunden übernommen wird. Daraus lässt sich der Vorteil für Unternehmen ableiten, durch Einbindung einer Crowd über eine Vermittlungsplattform Services rund um ihre Produkte anbieten zu können, für die keine Bezahlung in Form von Löhnen oder Sozialabgaben anfällt, da diese direkt von den Kunden übernommen wird.

Gleichzeitig kann dem Unternehmen dabei - wie bereits dargestellt - auch potenzieller Umsatz entgehen. Hinzu kommt, dass das Kompetenzniveau solcher Individuen - obwohl dieses durch die Crowdworking-Plattform im Vorfeld geprüft wird - in der Regel dennoch deutlich heterogener ist als dasjenige der nach einheitlichen Kriterien und Standards geschulten eigenen Beschäftigten. Der Einfluss auf und die Kontrolle über eigene Beschäftigte ist nicht zuletzt auf Grund des Direktionsrechts deutlich höher, ebenso die Sanktionierungs-Möglichkeiten bei Fehlverhalten. Auch kann der Einsatz externer Crowds zu Verunsicherung bei den internen Mitarbeitern bis hin zu im schlimmsten Fall Jobverlustängsten/inneren Kündigungen führen.

Information. Für das Erstellen von Produkten oder die Erbringung von Services werden Informationen genutzt. Im Fall der Swisscom Friends von Mila werden beispielsweise gute Kenntnisse der Produkte benötigt. Die Swisscom Friends von Mila sind meist selbst Kunden von Swisscom, haben sich also oft auch privat freiwillig für das jeweilige Produkt entschieden. Daraus lässt sich für Unternehmen folgender Vorteil ableiten: Der Einsatz solcher Crowds, die die Produkte des Unternehmens selbst nutzen, ohne dafür beispielsweise auf Grund der Tätigkeit als interne Mitarbeiter eine gewisse „Verpflichtung“ zu haben, bringt ein hohes Maß an Glaubwürdigkeit bei der Kommunikation von Informationen/Empfehlungen für das Unternehmen mit sich. Die Mitglieder der Crowd sind damit gute und sehr glaubwürdige Markenbotschafter.

Trotz dieses hohen Maßes an Glaubwürdigkeit verfügen externe Crowd Worker im Durchschnitt nicht über die gleichen Informationsquellen wie interne Mitarbeiter und damit oft auch nicht über gleich gute Kenntnisse der Produkte eines Unternehmens. Dieses kann sich negativ auf die Qualität der durch diese Crowd Worker im Auftrag des Unternehmens erbrachten Services und damit auf den Geschäftserfolg des Unternehmens auswirken.

Technologien. In der heutigen Arbeitswelt bilden Technologien meist die Basis für die Abwicklung von Prozessen und Aktivitäten. In diesem Fall sind das beispielsweise die Internetauftritte von Swisscom und Mila, über die Kunden Leistungen beauftragen können, oder die mobile Mila-App. Gemäß Einschätzung von Swisscom wird die Bedeutung digitaler Kanäle wie der über die Plattform Mila langfristig im Vergleich zu Call Centern oder physischen Point of Sale (POS)Kanälen vor Ort deutlich zunehmen. Mittels Mila kann Swisscom zudem eine Beschleunigung der Bearbeitung von Anfragen erreichen, da nun deutlich mehr potenzielle Bearbeiter zur Verfügung stehen und eine effiziente zentrale Steuerung der durch die Crowd erbrachten Services stattfindet. Daraus lässt sich der Vorteil ableiten, dass Unternehmen durch die Nutzung von auf Basis von Plattform-Technologien erbrachten Services Vorteile im Bereich der Beschleunigung der Abwicklung und der Effizienz der Steuerung des Arbeitssystems erzielen können.

Je nach Kundengruppe, Produkt und Unternehmen kann eine Abwicklung und Steuerung der Services durch solche Technologien aber auch nur die zweitbeste Lösung sein. So ist beispielsweise gerade bei technischen Unterstützungsleistungen wie bei Mila und Swisscom davon auszugehen, dass diese auch von vielen Kunden beauftragt werden, die weniger 
technikaffin sind (z.B. ältere Kunden). Gerade für diese Zielgruppe ist fraglich, ob beispielsweise die Beauftragung von Services per App der beste Zugangskanal ist.

\subsection{Fazit und Diskussion}

Die in diesem Beitrag erfolgte Exploration und Analyse der im deutschsprachigen Raum neuartigen Crowd Services-Plattform Mila und des Zusammenspieles mit deren Kunden und Eigentümer Swisscom AG liefert interessante Einblicke, die die Vor- und Nachteile dieses Modells sichtbar machen. Auf Basis dieser Vor- und Nachteile leiten wir nun im Folgenden aus unserer Sicht besonders essenzielle, konkrete Handlungsempfehlungen für Unternehmen, die Crowdworking-Plattform für die Vermittlung von Crowd Services nutzen möchten, ab:

Frühzeitig über den Einsatz von Crowd Services entscheiden. Die Anzahl der Unternehmen, die Crowdworking-Plattformen nutzt, steigt. Und gleichzeitig auch der dabei erzielte Umsatz: Die in Deutschland mit Sitz oder einem (physischen) Standort vertretenen Crowdworking-Plattformen konnten ihren Umsatz im Jahr 2016 im Vergleich zum Vorjahr im Durchschnitt um rund 90 Prozent erhöhen [4]. Das Prinzip der Erbringung von über Crowdworking-Plattformen beauftragten Services „von Kunden für Kunden“ scheint auf Grund der oben skizzierten Vorteile aktuell auch im deutschsprachigen Raum Zulauf zu bekommen: So hat am 17. Januar 2017 die Plattform „iAdvize“ mit Hauptsitz im französischen Nantes den Launch ihrer neuen Service-Plattform „ibbü“ (www.ibbu.com/de) für Deutschland bekannt gegeben, die Crowd Services für verschiedene Branchen vermittelt. Hält dieser Trend an und wollen immer mehr Unternehmen solche Crowd Services nutzen, dann kommt es nach Einschätzung der Autoren dieses Beitrages mittelfristig zu einer Knappheit an verfügbaren Crowd Workern. Es gilt daher für Unternehmen, frühzeitig über den Einsatz von Crowd Services zu entscheiden. Bei einer Entscheidung für einen solchen Einsatz kann eine ,First-Mover-Advantage‘ gegenüber Mitwettbewerbern erzielt sowie frühzeitig eine eigene Crowd aufgebaut und an sich gebunden werden.

Neue Kundenschnittstellen in die bisherigen Kanäle integrieren. Ist die Entscheidung gefallen, Crowd Services zu nutzen, gilt es, diese im Sinne einer effizienten Multikanalstrategie in die Gesamtheit der Vertriebskanäle des Unternehmens wie Apps, Call Center, Internet-Auftritt, Ladengeschäfte, u.v.m., sinnvoll zu integrieren. Für das Beispiel Swisscom haben wir in den obigen Abschnitten gezeigt, dass durch die Nutzung der Crowdworking-Plattform Mila weitere digitale Kundenzugangswege entstehen und sind auf die daraus resultierenden Vorteile eingegangen. Ein Grund für das erfolgreiche Zusammenspiel von Mila und Swisscom ist, dass je nach Bedarf verschiedene Kanäle und Beauftragungsmöglichkeiten für Services verfügbar sind [5]. So besteht beispielsweise auf dem Internet-Auftritt für Privatkunden von Swisscom die Möglichkeit, per Link direkt auf den Internet-Auftritt von Mila zu gehen und dort dann Services über Swisscom Friends zu beauftragen. Ein weiterer digitaler Zugangsweg resultiert aus der neuen Mila Mobile App. Eine enge Integration der verschiedenen („analogen“ und digitalen) Zugangswege hat zudem das Potenzial, den erwähnten Nachteil im Bereich "Corporate Identity“, der aus einem nicht als einheitlich wahrgenommenen Auftritt beim Kunden resultiert, zu begrenzen.

Externe mit internen Crowds (eigenen Mitarbeitern) eng verzahnen. Bei der Darstellung der Nachteile haben wir deutlich gemacht, dass durch den Einsatz externer Crowds in bereits bestehenden Vertriebsgebieten eine Verunsicherung der internen Mitarbeiter bis hin zu Jobverlustängsten stattfinden kann. Durch eine enge Verzahnung der externen Crowd Worker mit den internen Mitarbeitern eines Unternehmens können solche Verunsicherungen reduziert oder sogar weitgehend vermieden werden [9]. Insbesondere wenn deutlich wird, dass diese Crowd schwerpunktmäßig komplementäre Services bietet und bestehende Servicelücken im Angebot des Unternehmens schließt. Unternehmen sollten also Möglichkeiten einer engen Verzahnung externer und interner Crowds prüfen und damit ihre internen Mitarbeiter ,abholen:

Kontinuierlich in die Motivation der Crowd investieren. Die Erkenntnisse der Autoren dieses Beitrages im Rahmen ihrer allgemeinen Forschung zu Crowdworking-Plattformen (siehe u.a. [4, $10,11])$ zeigen eine große Herausforderung für diese Intermediäre: Eine engagierte, gut qualifizierte und hoch motivierte Crowd kontinuierlich zu gewährleisten und diese langfristig an 
sich zu binden. Ebenso wie Unternehmen Crowd Worker „on demand“ beauftragen können und für sie dabei im Gegensatz zu internen Mitarbeitern keine Fixkosten verbunden sind, können sich umgekehrt auch Crowd Worker ohne allzu große Wechselkosten (vom Verlust der auf einer Crowdworking-Plattform gewonnenen Reputation abgesehen) einer anderen Plattform beziehungsweise auf der gleichen Plattform den Aufträgen anderer Unternehmen zuwenden. Zu berücksichtigen ist zudem, dass eine große Anzahl an Crowd Workern auf den jeweiligen Plattformen inaktiv ist. Maßnahmen wie Prämien für besonders gute Beratungen, Reputationsmechanismen wie beispielsweise Sterne-Klassifizierungssysteme, die vorzeitige Bereitstellung neuer Produkte vor Markteinführung zu Testzwecken oder Umsatzbeteiligungen können helfen, die Loyalität der Crowd und ihre Motivation für die Erbringung von Services für das jeweilige Unternehmen zu erhalten oder zu steigern [10].

Die Crowd für eine Erweiterung des Vertriebsgebietes nutzen. Anhand des Beispiels Mila lässt sich zeigen, dass Unternehmen das eigene Vertriebsgebiet durch den Einsatz von Crowd Services schnell und kostengünstig erweitern können. Und dass Unternehmen damit auch in Regionen, in denen sie bisher nicht oder nur wenig präsent sind, mittels der Crowd Services erbringen lassen und den Vertrieb ihrer Produkte auch außerhalb des bisherigen Geschäftsgebietes unterstützen können. Insbesondere wenn die Strategie eines Unternehmens eine Expansion in kurzer Zeit vorsieht, sind Crowd Services damit eine kostengünstige und schnell implementierbare Alternative zum oft langwierigen und mühsamen Aufbau eines eigenen internen Vertriebs. Vor allem auch um erst einmal zu testen, wie die Produkte und Services in neuen potenziellen Vertriebsregionen ankommen, ist ein schneller Rollout von Services mittels des Einsatzes von Crowds gut geeignet. Gleichzeitig kann ebenso bei Bedarf ein zeitnaher kostengünstiger „Rollback“ erfolgen, wenn die Erfahrungen darauf hindeuten, dass die jeweilige Region für die eigenen Produkte und Services nicht Erfolg versprechend ist.

In diesem Beitrag haben wir anhand des Beispiels der Crowdworking-Plattform Mila und ihres Kunden Swisscom gezeigt, dass Crowd Services eine gute Ergänzung und Erweiterung des bestehenden Service-Angebotes eines Unternehmens darstellen können. Sie liegen im Trend der Plattform- und der Sharing-Economy und tragen der Tatsache Rechnung, dass Kunden einerseits immer öfter zu Produzenten von Services werden und andererseits bedarfsgerechte Services, auf Knopfdruck' erwarten. Crowd Services bieten zudem Chancen, den aus abnehmender Kundenloyalität und schnellen Wechseln zu anderen Anbietern entstehenden Herausforderungen zu begegnen. Gleichwohl es Maßnahmen bedarf, um potenziellen Nachteilen entgegenzuwirken, können aus dem Einsatz von Crowd Services erhebliche Vorteile in den Bereichen Effizienz, Geschwindigkeit, Kosten, Reichweite und Glaubwürdigkeit resultieren. Das Beispiel Mila (die durchschnittliche Bewertung beträgt nach Unternehmensangaben 4,8 von 5 möglichen Punkten und 92 Prozent der Kunden würden wieder beauftragen) zeigt zudem, dass die Zufriedenheit der Kunden auch bei Einsatz solcher Crowd Services sehr hoch sein kann. Der Trend der Nutzung der Crowd durch Unternehmen wird auch in Zukunft anhalten und sich auch auf immer komplexere Arbeit ausdehnen [11].

Die obigen Punkte stellen erste wichtige konkrete Handlungsempfehlungen für Unternehmen dar, die ihren Kundensupport verbessern, Wertschöpfung erhöhen und aus der Nutzung von Crowd Services mögliche Wettbewerbsvorteile generieren wollen. Crowdworking-Plattformen für die Erbringung von Supportleistungen der Art „von Kunden für Kunden“ bieten erhebliches Potenzial, das für verschiedene Arten von Arbeit innerhalb diverser Branchen genutzt werden kann.

Unternehmen, die potenziell von dem Einsatz solcher Crowd Services profitieren können und sich diesem Trend zu lange verschließen, riskieren nach Überzeugung der Autoren dieses Beitrages im Wettbewerb langfristig zurückzufallen.

\section{$1.6 \quad$ Danksagung}

Dieser Beitrag entstand im Rahmen des seitens des Bundesministeriums für Bildung und Forschung (BMBF) geförderten Projektes „Herausforderung Cloud und Crowd - Neue Organisationskonzepte für Dienstleistungen nachhaltig gestalten“ (Förderkennzeichen: 02K14A071, Projektträger: PTKA/Projektträger Karlsruhe). 


\subsection{Literatur}

[1] Brynjolfsson, E., McAfee, A.: Race against the machine. How the digital revolution is accelerating innovation, driving productivity, and irreversibly transforming employment and the economy. Digital Frontier Press, Lexington, Mass. (2011)

[2] Mrass, V., Li, M.M., Peters, C.: Towards a Taxonomy of Digital Work. 25. European Conference on Information Systems (ECIS), Guimarães, Portugal, 2017, 2515-2524 (2017)

[3] Kollmann, T., Schmidt, H.: Deutschland 4.0. Wie die Digitale Transformation gelingt. Springer Gabler, Wiesbaden (2016)

[4] Mrass, V., Peters, C.: Crowdworking-Plattformen in Deutschland. In: Leimeister, J. M. (Ed.), Working Paper Series, Nr. 16. Kassel (2017)

[5] Mrass, V., Peters, C., Leimeister, J.M.: Von Kunden für Kunden: Crowd Services als Erweiterung der Digital Customer Experience. HMD Praxis der Wirtschaftsinformatik 54, 821-837 (2017)

[6] Yin, R.K.: Case Study Research. Design and Methods. SAGE Publications, Inc., Los Angeles (2014)

[7] Alter, S.: Work System Theory: Overview of Core Concepts, Extensions, and Challenges for the Future. Journal of the Association for Information Systems (JAIS) 14, 72-121 (2013)

[8] Surowiecki, J.: The wisdom of crowds. Why the many are smarter than the few and how collective wisdom shapes business, economies, societies, and nations. Doubleday, New York (2004)

[9] Mrass, V., Peters, C., Leimeister, J.M.: One for All? Managing External and Internal Crowds through a Single Platform - A Case Study. 50th Hawaii International Conference on System Sciences (HICSS), Waikoloa, USA, 4324$4333(2017)$

[10] Mrass, V., Peters, C., Leimeister, J.M.: Managing Complex Work Systems via Crowdworking Platforms: How Deutsche Bank Explores AI Trends and the Future of Banking with Jovoto. 51th Hawaii International Conference on System Sciences (HICSS), Waikoloa, USA, 3391-3400 (2018)

[11] Mrass, V., Peters, C., Leimeister, J.M.: Managing Complex Work Systems via Crowdworking Platforms: The Case of Hamburger Hochbahn and Phantominds. 51th Hawaii International Conference on System Sciences (HICSS), Waikoloa, USA, 4112-4121 (2018) 